\title{
Characterization of the Recognition Specificity of BH2, a Monoclonal Antibody Prepared against the HLA-B27 Heavy Chain
}

\section{Hui-Chun Yu ${ }^{1,2, \dagger}$, Kuang-Yung Huang ${ }^{1,2,3, \dagger}$, Ming-Chi Lu ${ }^{2,3}$, Hsien-Lu Huang ${ }^{4}$, Wei-Ting Liu ${ }^{1}$, Wen-Chien Lee ${ }^{5}$, Su-Qin Liu ${ }^{2}$, Hsien-Bin Huang ${ }^{1, *}$ and Ning-Sheng Lai ${ }^{2,3, *}$}

1 Department of Life Science and Institute of Molecular Biology, National Chung Cheng University, Chia-Yi 621, Taiwan; E-Mails: df928039@tzuchi.com.tw (H.-C.Y.); hky0919@yahoo.com.tw (K.-Y.H.); weiting0608@hotmail.com (W.-T.L.)

2 Section of Allergy, Immunology and Rheumatology, Department of Medicine, Buddhist DaLin Tzu-Chi Hospital, Chia-Yi 622, Taiwan; E-Mails: dm252940@tzuchi.com.tw (M.-C.L.); df897226@tzuchi.com.tw (S.-Q.L.)

3 School of Medicine, Tzu-Chi University, Hualien 970, Taiwan

4 Department of Nutrition and Health Science, Fooyin University, Kaohsiung 831, Taiwan; E-Mail: estrus@mail2000.com.tw

5 Department of Chemical Engineering, National Chung Cheng University, Chia-Yi 621, Taiwan; E-Mail: chmwcl@ccu.edu.tw

$\dagger$ These authors contributed equally to this work.

* Authors to whom correspondence should be addressed;

E-Mails: biohbh@ccu.edu.tw (H.-B.H.); Q12015@tzuchi.com.tw (N.-S.L.);

Tel.: +886-5-272-0411 (ext. 53200) (H.-B.H.); +886-5-264-8000 (ext. 3223) (N.-S.L.);

Fax: +886-5-272-2871 (H.-B.H.); +886-5-264-8999 (N.-S.L.).

Academic Editor: Denis Girard

Received: 30 January 2015 / Accepted: 3 April 2015 / Published: 13 April 2015

Abstract: $\mathrm{BH} 2$, a monoclonal antibody prepared against the denatured human leukocytic antigen-B27 heavy chain (HLA-B27 HC), can immunoprecipitate the misfolded HLA-B27 $\mathrm{HC}$ complexed with Bip in the endoplasmic reticulum and recognize the homodimerized HLA-B27 HC that is often observed on the cell membrane of patients suffered from ankylosing spondylitis (AS). However, the recognition specificity of $\mathrm{BH} 2$ toward the other molecules of HLA-B type and toward the different types of HLA molecules remained 
uncharacterized. In this study, we carried out the HLA-typing by using the Luminex Technology to characterize the recognition specificity of $\mathrm{BH} 2$ and analyzed the binding domain of HLA-B27 $\mathrm{HC}$ by $\mathrm{BH} 2$. Our results indicated that $\mathrm{BH} 2$ preferably binds to molecules of HLA-B and -C rather than HLA-A and the binding site is located within the $\alpha 2$ domain of HLA-B27 HC.

Keywords: HLA-B27; ankylosing spondylitis; monoclonal antibody; HLA-typing

\section{Introduction}

The major histocompatibility complex (MHC) class I molecule is a heterodimeric protein that comprises a heavy chain (HC) and a light chain ( $\beta_{2}$-microglobulin, $\beta_{2}$ m). MHC class I molecules assemble with an antigenic peptide (approximate 8-12 amino acids in length) in the endoplasmic reticulum (ER) to form a heterotrimer and carry the bound peptide to the cell surface, where the peptide is displayed to cytotoxic T cells [1-4]. The HC (also called $\alpha$ chain), encoded by a human leukocytic antigen (HLA) gene, is polymorphic and is folded into three domains, $\alpha 1, \alpha 2$ and $\alpha 3$. Both $\alpha 1$ and $\alpha 2$ domains are associated together to form an antigen peptide-binding site, while the $\alpha 3$ domain containing a transmembrane segment is noncovalently associated with $\beta 2 \mathrm{~m}$ at the extracellular surface. MHC class I molecules are classified by three major types, HLA-A, HLA-B and HLA-C. Their genes are clustered on human chromosome 6 and their protein sequences share a high identity [5-8].

Ankylosing spondylitis (AS) is a chronic systemic inflammatory disease [9-13]. AS is highly linked with the expression of HLA-B27 [14,15]. Up to 95\% of AS patients are HLA-B27-positive. Several lines of evidence have demonstrated that the HLA-B27 heavy chain (HLA-B27 HC) has an intrinsic propensity to fold slowly in the ER before it is assembled with $\beta_{2} \mathrm{~m}$ and a peptide, in turn leading to the formation of disulfide-linked heavy-chain homodimers, (B27-HC) 2 [16-18]. (B27-HC) 2 is permitted to leave the ER, trafficked to the cell surface [16-18] and displayed to the natural killer-cell Ig-like receptor (KIR3DL2) as well as to T-helper 17 cells (Th17), in turn stimulating their activation and leading to one of the major pathogenic potentials in AS [19-21]. Thus, the levels of (B27-HC) 2 can be an index of the misfolded form of HLA-B27 HC. In our previous study, we have prepared an anti-HLA-B27 HC monoclonal antibody, BH2 [22]. The misfolded HLA-B27 can form a complex with Bip in the ER [10,23]. BH2 can immunoprecipitate the misfolded HLA-B27/Bip complex and recognize the disulfide-linked (B27-HC)2 [22]. However, the recognition specificity toward different HLA class I alleles by $\mathrm{BH} 2$ needed to be characterized. In this study, we characterize the binding specificity of $\mathrm{BH} 2$.

\section{Results}

\subsection{Recognition Specificity of BH2}

There are three major MHC class I gene clusters located on chromosome 6 of human, encoding HLA-A, HLA-B and HLA-C heavy chain molecules. DNA or protein sequences among these three different types of HLA molecules share a high identity. BH2 can recognize the misfolded HLA-B27 HC. However, the recognition specificity of $\mathrm{BH} 2$ toward the other HLA-B molecules or toward different 
types of HLA molecules remained unknown. Thus, we carried out HLA typing for BH2 by using the Luminex Technology to analyze its recognition specificity. Each individual microbead was conjugated with the unique HLA molecule antigen. The mixtures of microbeads consisting of HLA molecules were incubated with $\mathrm{BH} 2$, reacted with R-phycoerythrin-conjugated secondary antibodies and then analyzed by flow cytometry. Value of mean fluorescence intensity (MFI) for different types of HLA antigens bound by $\mathrm{BH} 2$ is shown in Figure 1A. Cut-off values of MFI for HLA molecules were set at 637. MFI value more than 637 was considered as a positive binding of HLA molecule by BH2. The results show that $\mathrm{BH} 2$ binds to HLA-B and HLA-C molecules. However, $\mathrm{BH} 2$ only binds to HLA-A11, but not to other HLA-A antigens in our assay. HLA-B, -C, and -A molecules recognized by BH2 are summarized in Table 1. In addition, $\mathrm{BH} 2$ binding to $\mathrm{MHC}$ class II molecules were also analyzed by the same technique using microbeads coated with MHC class II antigens. Value of MFI for each type of MHC class II molecules are shown in Figure 1B. Cut-off values of MFI for this assay were set at 500. MFI value of more than 500 was suggested to be a positive binding between $\mathrm{BH} 2$ and $\mathrm{MHC}$ class II molecule. All of MFI values are less than 500, suggesting that $\mathrm{BH} 2$ cannot recognize MHC class II molecules.

(A)

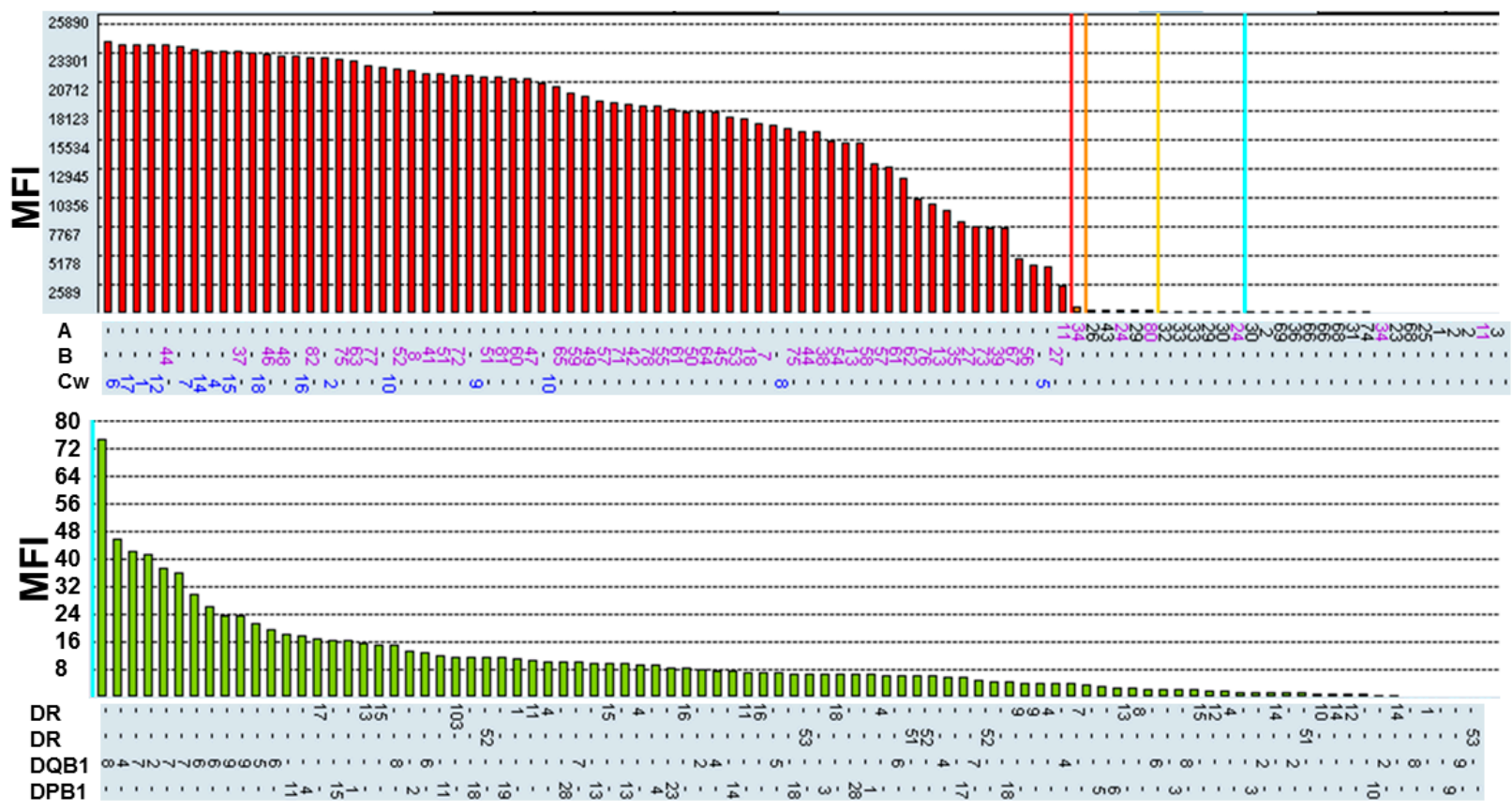

Figure 1. Characterization of binding specificity for $\mathrm{BH} 2$ by human leukocytic antigen (HLA)-typing. (A) Analysis of BH2 binding to major histocompatibility complex (MHC) class I molecules by using the Luminex Technology. MFI represents mean fluorescence intensity. Cut-off values of mean fluorescence intensity (MFI) for positive binding were set at 637; (B) Analysis of BH2 binding to MHC class II molecules by using the Luminex Technology. Cut-off values of MFI for positive binding were set at 500 . 
Table 1. MHC class I molecules, not class II molecules, bind to BH2. The results were summarized from Figure 1A,B.

\begin{tabular}{cc}
\hline Class I (Positive) & Class II (Positive) \\
\hline Cw10, B27, B57, B51, B61, B13, B44 & \\
B75, B55, Cw4, Cw12, B49, B38, Cw1 & \\
B76, B54, Cw16, B8, B48, B35, Cw15 & \\
B42, Cw7, Cw9, B41, B7, B62, Cw6 & \\
\hline B82, B37, Cw17, B60, B56, B65, B45, B81 & Negative \\
B59, B52, B39, Cw2, Cw14, B78, B46 & \\
Cw5, Cw8, B72, B58, B63, B77, B73 & \\
B71, B64, B53, B67, B47, B18, Cw18 & \\
B50, A11 & \\
\hline
\end{tabular}

\subsection{BH2 Binds to the a2 Domain of HLA-B2704 HC}

The heavy chain of MHC class I molecules from $N$ - to $C$-terminus consists of three domains, $\alpha 1, \alpha 2$ and $\alpha 3$, each about 90 residues in length. Both $\alpha 1$ and $\alpha 2$ domains fold into a module to form an antigenic peptide binding site. The $\alpha 3$ domain connected to the transmembrane domain via a short flexible linker is a site for the non-covalent interaction with $\beta_{2} \mathrm{~m}$ at the extracellular surface. We also analyzed which domain of HLA-B27 HC is recognized by BH2. Each domain of HLA-B2704 HC was cloned and over-expressed in E. coli (BL21 DE3). Figure 2A indicates the expression of each domain induced by isopropyl $\beta$-D-1-thiogalactopyranoside (IPTG). Western blotting analysis demonstrated that $\mathrm{BH} 2$ binds to the $\alpha 2$ domain (Figure 2B), but not to $\alpha 1$ and $\alpha 3$ domains. The recombinant $\alpha 2$ domain of HLA-B27 HC showing multiple bands on SDS-PAGE (Figure 2B) may arise from formation of the inclusion body or from the aggregated forms. We randomly picked up some $\alpha 2$ domain of HLA class I alleles for sequence alignment analysis to figure out the potential binding epitope by BH2 (Figure 3). BH2 recognizes HLA-B27, -B41, -B15, -Cw1, -Cw6, -Cw12 and -A11, but not HLA-A2. Based on the sequence alignment of $\alpha 2$ domains, we hypothesized that Pro-129 and Gly-131 of $\alpha 2$ domain could play a critical role in BH2 binding. However, after replacing Pro-129 and Gly-131 of $\alpha 2$ domain in HLA-B27 by Ser and Trp, respectively, using site-directed mutagenesis. The mutant $\alpha 2$ domain of HLA-B27 remained bound to $\mathrm{BH} 2$, seen by western blotting assay (Figure 4). HC10 is one of the monoclonal antibodies that were prepared against a mixture of denatured HLA-B7 and -B40 heavy chains [23]. HC10 can immunoprecipitate the misfolded HLA-B27/Bip complex [10,24] and recognize the homodimeric HLA-B27, (B27-HC)2 [24]. Although it is still unknown which of the $\alpha$ domains of HLA-B27 HC is recognized by HC10, HC10 prefers to bind to HLA-B and HLA-C types than to HLA-A type [24]. Both BH2 and HC10 can recognize the misfolded HLA-B27 HC, but their binding specificity toward HLA-A loci is subtly different. HC10 recognizes HLA-A3 and -A33 [23]. However, in our HLA-typing, BH2 fails to recognize HLA-A3 and -A33. Up to now, only $\mathrm{HC} 10$ and $\mathrm{BH} 2$ have been proved to recognize the misfolded HLA-B27 HC and (B27-HC)2. 

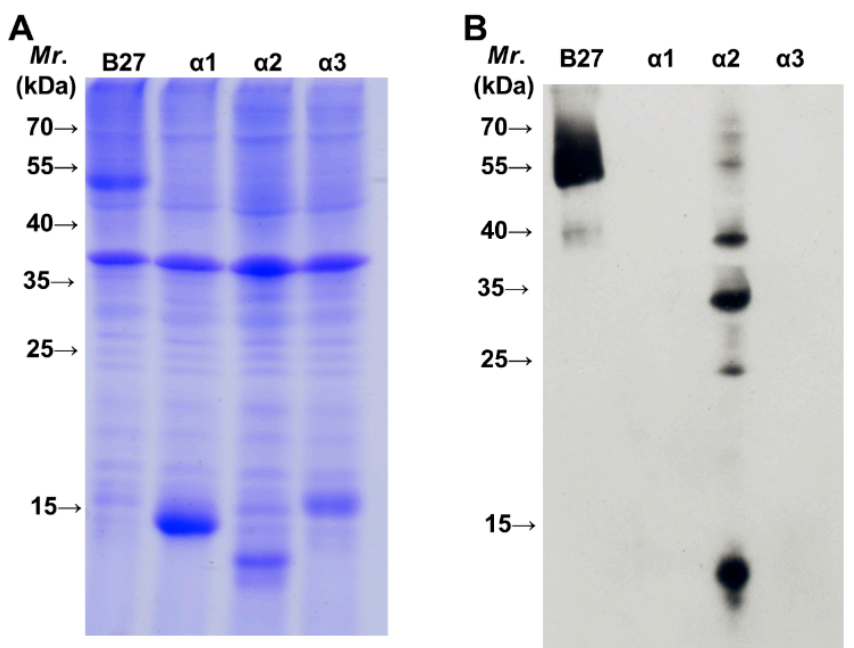

Figure 2. Analysis of HLA-B27 heavy chain domain recognized by BH2. (A) SDS-PAGE analysis of HLA-B27 domains overexpressed in E. coli (BL21 DE3); (B) Domain of HLA-B27 heavy chain recognized by $\mathrm{BH} 2$ was analyzed by western blotting. An aliquot $(20 \mu \mathrm{g})$ of each crude protein extracted from E. coli bacteria that have overexpressed the indicated domain of HLA-B27 HC was separated by SDS-PAGE (15\%) and analyzed by western blotting using BH2 monoclonal antibody.

\begin{tabular}{|c|c|}
\hline 27 & SCDVGPDGRLL \\
\hline HLA & YYGCDVGPDGRLLRGHNYYYYGKDYIALNEDLRSWTAA \\
\hline 8 & GSHI IQRMYGCDLGPDGRLLRGHDQSAYDGKDY \\
\hline 60 & GSHTLQRMYGCDV \\
\hline & GSH \\
\hline HLA-Cw6 & GSHTLQWMYGCDLGPDGRLLRGYDQSAYDGKDYIALNEDLRSWTAA \\
\hline $\mathrm{CA}-\mathrm{Cw} 12$ & GSHTLQRMYGCDLGPDGRLLRGYDQVAYDGKDYIALNEDLRSWTAA \\
\hline CA-A11 & GSHTIQIMYGCDVGPDGRFLRGYRQDAYDGKDYIALNEDLRSWTAA \\
\hline LA-A2 & RMYGCDVGSDWRFLRGYHQYAYDGKDYIALKEDLRSWTAA \\
\hline HLA-B27 & EQLRAYLEGECVEWLRRYLENGKETLQRA² \\
\hline HLA-B41 & EQDRAYLEGTCVEWLRRYLENGKDTLERA \\
\hline HLA-B58 & TAAQITQRKWEAARVAEQLRAYLEGLCVEWLRRYLENGKETLQRA \\
\hline HLA-B60 & TAAQISQRKLEAARVAEQLRAYLEGE \\
\hline HLA- & FWLRRYLENGKESLQRA \\
\hline HLA- & WWRAYLEGTCVEWLRRYLENGKETLQRA \\
\hline HLA-Cw12 & QWRAYLEGTCVEWLRRYLENGKETLQRA \\
\hline$A-A 11$ & QQRAYLEGRCVEWLRRYLENGKETLQRT \\
\hline & AYLEGTCVEWLR \\
\hline
\end{tabular}

Figure 3. Amino acid sequence alignment of HLA-B27 $\mathrm{HC} \alpha 2$ domain with that of the indicated HLA-B, -C, and -A molecules. BH2 binds to HLA-B27, -B41, -B58, -B60, -Cw1, -Cw6, -Cw12 and -A11, but not to HLA-A2 in HLA-typing assay. The non-consensus residues compared with HLA-B27 are marked with red. Double replacements of Pro-129 and Gly-131 of HLA-B27 HC with Ser and Trp, respectively, are marked as green. 


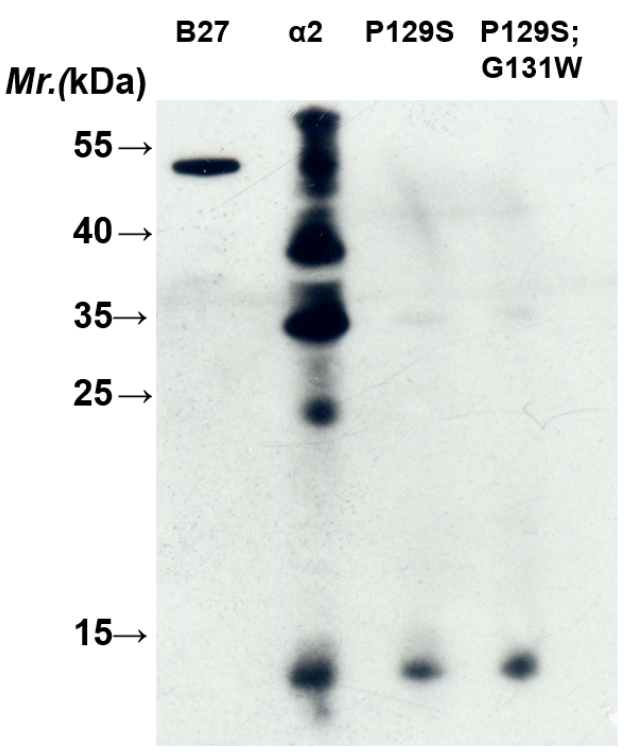

Figure 4. Double replacements of Pro-129 and Gly-131 with Ser and Trp, respectively, on the $\alpha 2$ domain do not affect the BH2-binding. An aliquot $(20 \mu \mathrm{g})$ of each crude protein extracted from $E$. coli bacteria that have overexpressed the indicated mutant $\alpha 2$ domain of HLA-B27 HC was separated by SDS-PAGE (15\%) and analyzed by western blotting using BH2 monoclonal antibody.

\section{Experimental Section}

\subsection{Materials}

Dithiothreitol (DTT), Tris, Luria-Bertani (LB) broth, kanamycin, isopropyl $\beta$-D-1-thiogalactopyranoside (IPTG), sodium dodecyl sulfate (SDS), TEMED, ammonium persulfate, acrylamide, glycine and Tris Base were obtained from Sigma-Aldrich (St. Louis, MO, USA).

\subsection{HLA-Typing}

HLA recognition specificity of $\mathrm{BH} 2$ was characterized following the methods as described by the manufacturer (Luminex Corporation, Austin, TX, USA) [25]. Briefly, $10 \mu \mathrm{L}$ of LABScreen Mixed kit (One Lambda, Canoga Park, CA, USA) containing microbeads coated with purified Class I or Class II HLA antigens were incubated with $30 \mu \mathrm{L}$ of BH2 monoclonal antibody in the dark at room temperature for $30 \mathrm{~min}$. All components were washed with the buffer to remove the unbound BH2. The antibody bound to the antigen coated on the microbeads was reacted with $R$-phycoerythrin-conjugated goat anti-mouse IgG. The fluorescent emission on the microbeads was detected by the LABScan 100 flow analyzer (Canoga Park, CA, USA) and analyzed by One Lambda software (Canoga Park, CA, USA). Cut-off values were established according to the manufacturer's instructions.

\subsection{Analysis of BH2-Binding Domain}

HLA-B27 HC consists of three domains ( $\alpha 1, \alpha 2$ and $\alpha 3)$. The full-length of human HLA-B27 HC cDNA was served as a template and cDNA of each domain was cloned by PCR using the following primers: 
Alpha 1 primers: 5'-GGCGGCTCCCACTCCATGAGG-3' and 5'-GGCCTCGCTCTGGTTGATG-3' Alpha 2 primers: 5'-GGGTCTCACACCCTCCAGAA-3' and 5'-CGCGCGCTGCAGCGTCTC-3' Alpha 3 primers: 5'-GACCCCCCAAAGACACACG-3' and 5'-CCATCTCAGGGTGAGGGG-3'

The resulting product was cleaved by BamHI/EcoRI and subcloned into the pET28a plasmid. E. coli (BL21 DE3) cells transformed with the recombinant vector encoding $\alpha 1, \alpha 2$ or $\alpha 3$ domain of B27 HC were grown in $5 \mathrm{~mL}$ of LB broth at $37^{\circ} \mathrm{C}$ for $3 \mathrm{~h}$. Then, protein expression was induced by IPTG (final concentration of $1 \mathrm{mM}$ ) for $3 \mathrm{~h}$. Bacteria $(1 \mathrm{~mL})$ were subjected to centrifugation at $12,000 \times \mathrm{g}$ for $3 \mathrm{~min}$ and the supernatant was discarded. The pelleted cells were re-suspended by $100 \mu \mathrm{L}$ of $1 \%$ SDS and ruptured by ultrasonication. An aliquot $(20 \mu \mathrm{L})$ of extracted proteins was separated by SDS-PAGE $(15 \%)$ and analyzed by western blotting using $\mathrm{BH} 2$ monoclonal antibody.

\subsection{Site-Directed Mutagenesis}

Site-directed mutagenesis was carried out by using the QuikChange Site-directed Mutagenesis Kit (Stratagene, La Jolla, CA, USA) with the primers ( $\alpha 2$ domain P129S: 5'-GGCTGCGACGTGGGG TCGGACGGGCGCCTCCTCCGC-3'; $\alpha 2$ domain P129S; G131W: 5'-GGCTGCGACGTGGGGTCG GACTGGCGCCTCCTCCGCGGG-3') following the methods described by the manufacturer. The plasmids, pET28a- $\alpha 2$ domain and pET28a- $\alpha 2$ domain P129S, were used as the templates for site-directed mutagenesis to produce the plasmids, pET28a- $\alpha 2$ domain P129S and pET28a- $\alpha 2$ domain P129S; G131W, respectively.

\section{Conclusions}

We have demonstrated that $\mathrm{BH} 2$ prefers to bind to HLA-B and HLA-C rather than to HLA-A types in HLA typing assay (Figure 1A). BH2 binds to HLA-A11, but show a more weak binding affinity. No other HLA-A molecules were observed to interact with BH2 in our assays (Figure 1A). BH2 binds to the $\alpha 2$ domain of HLA-B27. However, the recognition site in $\alpha 2$ domain of HLA-B27 HC by BH2 needs to be characterized.

\section{Acknowledgments}

This work was supported by grants from the Buddhist DaLin Tzu Chi Hospital (SP98-2-3 and DTCRD 103-E-14) and from the Ministry of Economic Affairs (103-EC-17-A-09-S1-206).

\section{Author Contributions}

Hui-Chun Yu, Kuang-Yung Huang, Su-Qin Liu and Wei-Ting Liu performed the experiments. Ming-Chi Lu, Hsien-Lu Huang and Wen-Chien Lee contributed data analysis. Hui-Chun Yu, Kuang-Yung Huang, Hsien-Bin Huang and Ning-Sheng Lai designed the experiments. Hsien-Bin Huang and Ning-Sheng Lai wrote the manuscript.

\section{Conflicts of Interest}

The authors declare no conflict of interest. 


\section{References}

1. Ploegh, H.L.; Orr, H.T.; Strominger, J.L. Major histocompatibility antigens: The human (HLA-A, -B, -C) and murine (H-2K, H-2D) class I molecules. Cell 1981, 24, 287-299.

2. Rammensee, H.G.; Falk, K.; Rötzschke, O. Peptide naturally presented by MHC class I molecules. Annu. Rev. Immunol. 1993, 11, 213-244.

3. Androlewicz, M.J.; Ortmann, B.; van Endert, P.M.; Spies, T.; Cresswell, P. Characteristics of peptide and major histocompatibility complex class I/ $\beta 2$-microglobulin binding to the transporters associated with antigen processing (TAP1 and TAP2). Proc. Natl. Acad. Sci. USA 1994, 91, 12716-12720.

4. Ritz, U.; Seliger, B. The transporter associated with antigen processing (TAP): Structural integrity, expression, function, and its clinical relevance. Mol. Med. 2001, 7, 149-158.

5. Jones, E. MHC class I and class II structures. Curr. Opin. Immunol. 1997, 9, 75-79.

6. Garboczi, D.N.; Ghosh, P.; Utz, U.; Fan, Q.R.; Biddison, W.E.; Wiley, D.C. Structure of the complex between human T-cell receptor, viral peptide and HLA-A2. Nature 1996, 384, 134-141.

7. Kostyu, D.D.; Hannick, L.I.; Traweek, J.L.; Ghanayem, M.; Heilpern, D.; Dawson, D.V. HLA class I polymorphism: Structure and function and still questions. Hum. Immunol. 1997, 57, 1-18.

8. Pamer, E.; Cresswell, P. Mechanisms of MHC class I-restricted antigen processing. Annu. Rev. Immunol. 1998, 16, 323-358.

9. Colbert, R.A. HLA-B27 misfolding: A solution to the spondyloarthropathy conundrum? Mol. Med. Today 2000, 6, 224-229.

10. Colbert, R.A.; DeLay, M.L.; Klenk, E.I.; Layh-Schmitt, G. From HLA-B27 to spondyloarthritis: A journey through the ER. Immunol. Rev. 2010, 233, 181-202.

11. Melis, L.; Elewaut, D. Immunopathogenesis of spondykoarthritis: Which cells drive disease? Arthritis Res. Ther. 2009, 11, 233-238.

12. Smith, J.A.; Marker-Hermann, E.; Colbert, R.A. Pathogenesis of ankylosing spondylitis: Current concepts. Best Pract. Res. Clin. Rheumatol. 2006, 20, 571-591.

13. Tam, L.S.; Gu, J.; Yu, D. Pathogenesis of ankylosing spondylitis. Nat. Rev. Rheumatol. 2010, 6, 399-405.

14. Caffrey, M.F.; James, D.C. Human lymphocyte antigen association in ankylosing spondylitis. Nature 1973, 242, 121.

15. Brewerton, D.A.; Hart, F.D.; Nicholls, A.; Caffrey, M.; James, D.C.O.; Sturrock, R.D. Ankylosing spondylitis and HLA-B27. Lancet 1973, 1, 904-907.

16. Allen, R.L.; O’Callaghan, C.A.; McMichael, A.J.; Bowness, P. HLA-B27 can form a novel 32-microglobulin-free heavy chain homodimer structure. J. Immunol. 1999, 62, 5045-5048.

17. Kollnberger, S.; Bird, L.; Sun, M.Y.; Retiere, C.; Braud, V.M.; McMichael, A.; Bowness, P. Cell-surface expression and immune receptor recognition of HLA-B27 homodimers. Arthritis Rheumatol. 2002, 6, 2972-2982.

18. Dangoria, N.; DeLay, M.L.; Kingsbury, D.J.; Mear, J.P.; Uchanska-Ziegler, B.; Ziegler, A; Colbert, R.A. HLA-B27 misfolding is associated with aberrant intermolecular disulfide bond formation (dimerization) in the endoplasmic reticulum. J. Biol. Chem. 2002, 77, 23459-23468. 
19. Kollnberger, S.; Bird, L.A.; Roddis, M.; Hacquard-Bouder, C.; Kubagawa, H.; Bodmer, H.C.; Breban, M.; McMichael, A.J.; Bowness, P. HLA-B27 heavy chain homodimers are expressed in HLA-B27 transgenic rodent models of spondyloarthritis and are ligands for paired Ig-like receptors. J. Immunol. 2004, 73, 1699-1710.

20. Chan, A.T.; Kollnberger, S.D.; Wedderburn, L.R.; Bowness, P. Expansion and enchanced survival of natural killer cells expressing the killer immunoglobulin-like receptor KIR3DL2 inspondylarthritis. Arthritis. Rheumatol. 2005, 52, 3586-3595.

21. Bowness, P.; Ridley, A.; Shaw, J.; Chan, A.T.; Wong-Baeza, I.; Fleming, M.; Cummings, F.; McMichael, A.; Kollnberger, S. Th17 cells expressing KIR3DL2+ and responsive to HLA-B27 homodimers are increased in ankylosing spondylitis. J. Immunol. 2011, 186, 2672-2680.

22. Yu, H.C.; Lu, M.C.; Li, C.; Huang, H.L.; Huang, K.Y.; Liu, S.Q.; Lai, N.S.; Huang, H.B. Targeted delivery of an antigenic peptide to the endoplasmic reticulum: application for development of a peptide therapy for ankylosing spondylitis. PLOS ONE 2013, 8, e77451.

23. Stam, N.J.; Spits, H.; Ploegh, H.L. Monoclonal antibodies raised against denatured HLA-B locus heavy chains permit biochemical characterization of certain HLA-C locus products. J. Immunol. 1986, 137, 2299-2306.

24. Tran, T.M.; Satumtira, N.; Dorris, M.L.; May, E.; Wang, A.; Furuta, E.; Taurog, J.D. HLAB27 in transgenic rats forms disulfide-linked heavy chain oligomers and multimers that bind to the chaperone BiP. J. Immunol. 2004, 172, 5110-5119.

25. Colombo, M.B.; Haworth, S.E.; Poli, F.; Nocco, A.; Puglisi, G.; Innocente, A.; Serafini, M.; Messa, P.; Mario, M. Luminex technology for anti-HLA antibody screening: Evaluation of performance and of impact on laboratory routine. Cytometry 2007, 72B, 465-471.

(C) 2015 by the authors; licensee MDPI, Basel, Switzerland. This article is an open access article distributed under the terms and conditions of the Creative Commons Attribution license (http://creativecommons.org/licenses/by/4.0/). 